TP Periodica Polytechnica

Transportation Engineering

43(1), pp. 22-26, 2015

DOI: 10.3311/PPtr.7481

Creative Commons Attribution (i)

RESEARCH ARTICLE

\section{Bus Drivers Risk Perception of Roadway Hazards}

\author{
Evangelos MITSAKIS ${ }^{1 *}$, Iraklis STAMOS ${ }^{1}$, \\ Josep Maria SALANOVA GRAU ${ }^{1}$, Maria MORFOULAKI ${ }^{1}$
}

ReCEIVEd 16 April 2014, ACCePted AfTeR REVISION 4 MAY 2014

\begin{abstract}
This paper presents a risk assessment algorithm that can be integrated within advanced safety monitoring systems of urban road networks. The research conducted is part of the MISS (Monitor Integrated Safety System) research project funded under the 6th Framework Program of the European Union. MISS aims to develop an innovative platform to dynamically sense and predict natural and infrastructure conditions, so that safety and efficiency of transport operations in multienvironmental scenarios are improved. The platform enables intelligent exchange of structured information between operational fleet vehicles and a Unified Operative Centre where information is elaborated and actions are planned. As part of the MISS program, efficient off- and online risk assessment methods have been designed to enable public authorities to better monitor and respond to hazardous incidents. These have been based on the analysis of stated preference questionnaires that have been filled in by expert bus drivers of the Bologna public transport agency.
\end{abstract}

\section{Keywords}

road safety, bus drivers, risk assessment

${ }^{1}$ Centre for Research and Technology Hellas, Hellenic Institute of Transport, Thessaloniki, Greece

*Corresponding author, e-mail: emit@certh.gr

\section{Introduction}

This paper aims to provide responsive measures to various combinations of weather, light, road type and road/traffic conditions which occur during the daily transport service operations of ATC buses on bus lanes in the Province of Bologna, Italy. Each combination is assigned to a response measure according to the risk level, as this has been stated by experts. The data has been gathered from questionnaires, which have been answered by expert panels comprised of ATC bus drivers. The final risk index, in form of responsive measures, is derived from a non-parametric statistical analysis of the questionnaires results. The pseudo-code for the risk assessment algorithm has also been written and is presented in the paper. The questionnaires have been designed in a form which has been considered most suitable both from the perspective of the possibilities for the analysis of the results, as well as from the perspective of motivating the experts to answer each question carefully (since the questionnaire has been comprised of 64 questions, each having five possible answers) by relating the risk associated to each described situation to the measure that should be taken in order to eliminate the related risk. It is believed that the questionnaire with answers relating to exact measures rather than to numerical risk indexes provided a motivation to the experts to consider their answers more, since they can realize more easily that their own safety is directly concerned. Their knowledge of the exact conditions of the road network and the associated risks that they are exposed to enables the development of a robust risk assessment algorithm. The implementation of the risk assessment algorithm aims to decrease the probability of an event (accident) by enabling preventive measures, either directly related to a possible accident or indirectly leading to a high risk situation that can lead to an accident.

\section{Literature review}

The literature review for road accidents involving buses or coaches and their associated risks has identified a gap in this study area. However, there is a certain amount of published research that has been reviewed. Albertson and Falkmer [1] 
have identified patterns in bus and coach related accidents and have suggested possible future measures for improvement of bus and coach safety. Their results form a multi-faceted pattern which indicates that despite the fact that fatalities were more frequent on rural roads, a vast majority of all bus and coach casualties occurred on urban roads and in dry weather conditions. Boarding and alighting caused about one-third of all injury cases. Collisions were a major injury-contributing factor. Buses and coaches most frequently collided with cars, but unprotected road users were hit in about one-third of all cases of a collision, the point of impact on the bus or the coach being typically frontal or side. Rollovers occurred in almost all cases of severe coach crashes. Side contacts are found to be the most common type of accident for public buses in the city of Uppsala case study of Wahlberg, who also indicates that more than $25 \%$ of all public bus accidents occur near or at bus stops [2]. In his second publication [3] Wahlberg has further analyzed public bus accidents by further subcategorizing them and the results have shown that injuries were common in intersection accidents, that bus stops present larger risk for side contacts, while single vehicle accidents were seldom preceded by the loss of control or a skid. Risky behavior of both public bus commuters and public bus drivers has been analyzed in a case study of Mirza, Mirza, Chotani and Luby [4] for the city of Karachi. The results of their study showed that $33 \%$ of the bus commuters did not wait for the bus to stop, 54\% stepped off in the center of the road and $84 \%$ did not look out for traffic. Among the embarking commuters, 38\% got on moving buses, $73 \%$ climbed on buses filled to their outer foot boards and $83 \%$ waited for buses on the street. Males were more likely than females to jump off a moving bus ( $43 \%$ versus $1.6 \%, \mathrm{P}<0.001)$, get on a moving bus ( $49 \%$ versus $12 \%, \mathrm{P}<0.001)$, and run to catch a bus $(45 \%$ versus $8 \%, \mathrm{P}<0.001)$. At the bus stops, $30 \%$ of the buses did not stop completely, $46 \%$ stopped away from the stop and $79 \%$ stopped in the center of the road. Where traffic police were present buses were more likely to race $(9 \%$ versus $3 \%, \mathrm{P}<0.05)$ and to cut off other vehicles $(13 \%$ versus $2 \%, \mathrm{P}<0.001)$ than where police were absent. Traditional efforts to regulate bus traffic through traffic police is reported as ineffective.

\section{Methodology}

A systematic questionnaire of the perception of potential risks and hazards by bus drivers in Bologna has been conducted. The developed questionnaire aims to analyze the importance of the main accident risk parameters (type of roadway, environmental conditions, type of lane-blocking object etc.) with a direct correlation to the perceived level of risk (by the bus drivers) and the countermeasures that should be deployed in each case. The questionnaire which has been designed to be answered by the ATC experts (bus drivers) consists of 65 questions. 64 out of the 65 questions have 5 possible answers. The questions are based on combinations of the following parameters: weather conditions, light conditions, road type and traffic event (in form of a bus lane blocking event) which corresponds to a significant risk level due to the disruption that is resulted through it to the bus transport service operations. The parameters are categorized as follows:

- Weather conditions - GOOD or BAD /

- Light conditions - DAYLIGHT or NIGHTLIGHT

- Road type - ONE WAY ROAD or TWO WAY ROAD

- Lane blocking event - VEHICLE or OBSTACLE or IMPROPERLY MARKED / CONSTRUCTION ZONE and FULLY BLOCKING BUS LANE or PARTLY BLOCKING BUS LANE and ALARM LIGHTS ON or ALARM LIGHTS OFF.

The parameters to be included in the questionnaire have been chosen after taking into consideration the following:

- Simplicity of the final algorithm that will be integrated into MISS, as the input data will be related to the data (parameters) that have been included in the questionnaire. The bus drivers will have to provide the algorithm with input only for the four parameters selected.

- The parameters which have been chosen are related to bus transport operations on bus lanes and not all other transport means.

- The size of the questionnaire had to be kept in a reasonable size in order to help the participants be as accurate as possible.

- The literature review and the accident reasons that have been identified as most common.

All the possible combinations of the above parameters provide the questionnaire with 64 different questions.

The possible answers to the above questionnaire are as follows:

- IMMEDIATELY TOW

- TOW BUT NOT URGENT

- TICKET

- WARNING

- IGNORE (DO NOTHING)

One extra question provides the experts with the opportunity to describe on their own words two or more situations or combinations of events that have not been captured through the questionnaire but based on their expertise has a significant level of risk and according to their opinion endangers the bus transport operations on bus lanes. The answers of the experts to the questions have been transformed from text string values to numerical values. The transformation has occurred according to the Table 1 . 
Table 1 Transformation of text string to numerical values

\begin{tabular}{cc}
\hline Measure & Value \\
\hline Immadiately tow & 1 \\
Tow but not urgent & 2 \\
Ticket & 3 \\
Warning & 4 \\
Ignore & 5 \\
No answer & blank \\
\hline
\end{tabular}

\section{Analysis and results}

The results of the questionnaire consisted of responses of 11 experts. The steps of the analysis are:

\section{Mean value analysis}

There are several statistical quantities called means, e.g., harmonic mean, geometric mean, arithmeticgeometric mean, and root-mean-square. When applied to two elements $\mathrm{a}$ and $\mathrm{b}$ with $0<\mathrm{a} \leq \mathrm{b}$, these means satisfy $\mathrm{H}(\mathrm{a}, \mathrm{b}) \leq \mathrm{G}(\mathrm{a}, \mathrm{b}) \leq \mathrm{AGM}$ $(\mathrm{a}, \mathrm{b}) \leq \mathrm{A}(\mathrm{a}, \mathrm{b}) \leq \mathrm{RMS}(\mathrm{a}, \mathrm{b})$

The quantity commonly referred to as "the" mean of a set of values is the arithmetic mean

$$
x=\left(\frac{1}{n}\right) \sum_{i=1}^{n} X_{i}
$$

also called the (unweighted) average. For the analysis of the questionnaires mean values (averages) have been calculated both for the each questionnaire independently and for each question.

\section{Wilcoxon Non-parametric Test}

The Wilcoxon signed-rank test can be applied to paired data. Let $(X 1 j, X 2 j), j=1,2, n$ be a collection of paired observations from two continuous distributions that differ only with respect to their means. (It is not necessary that the distributions of $\mathrm{X} 1$ andX2 be symmetric.) This assures that the distribution of the differences $\mathrm{Dj}=\mathrm{X} 1 \mathrm{j}-\mathrm{X} 2 \mathrm{j}$ is continuous and symmetric. Thus, he null hypothesis is $\mathrm{H} 0: \mu 1=\mu 2$, which is equivalent to $\mathrm{H} 0: \mu \mathrm{D}=0$. We initially consider the two-sided alternative H1: $\mu 1 \neq \mu 2$ (or H1: $\mu \mathrm{D} \neq 0$ ). To use the Wilcoxon signedrank test, the differences are first ranked in ascending order of their absolute values, and then the ranks are given the signs of the differences. Ties are assigned average ranks. Let $\mathrm{W}+$ be the sum of the positive ranks and Wbe the absolute value of the sum of the negative ranks, and $\mathrm{W}=\min (\mathrm{W}+, \mathrm{W}-)$. If the observed value $\mathrm{w} \leq \mathrm{W}^{*} \alpha$, the null hypothesis $\mathrm{H} 0: \mu 1=\mu 2$ (or $\mathrm{H} 0: \mu \mathrm{D}=0)$ is rejected. For one-sided tests, if the alternative is $\mathrm{H} 1: \mu 1>\mu 2$ (or $\mathrm{H} 1: \mu \mathrm{D}>0$ ), reject $\mathrm{H} 0$ if $\mathrm{w}-\leq \mathrm{w}^{*} \alpha$ and if $\mathrm{H} 1: \mu 1<\mu 2$ (or $\mathrm{H} 1: \mu \mathrm{D}<0$ ), reject $\mathrm{H} 0$ if $\mathrm{w}^{+} \leq \mathrm{w}^{*} \alpha$. For the analysis of the questionnaire, the Wilcoxon non-parametric test has been applied in four paired data sets. The first includes the data pairs of all questions with the order that they appear in the questionnaire, the second includes the data pairs according to light conditions, that is day or night, the third includes the data pairs according to road type, that is one- or two-way road and the fourth includes that data pairs according to weather conditions, that is good or bad weather The Wilcoxon nonparametric produces values of significance, which show for every pair of data that is being examined, whether they it is significantly different or not, according to the significance level that has been chosen $(95 \%)$.

Levels of significance for the Wilcoxon Non-parametric Test The level of significance has been set to ,05 (95\%). According to this level of significance, the following question pairs for the four combinations have been found to have a significance value lower than 0,05, which means that the null hypothesis (the two questions in each pair have no significant difference) is rejected and thus there is significant difference between the answers of the experts to these question pairs.

Table 2 Question pairs with level of significance lower than 0,05 for pairs of data with the order of appearance in the questionnaire.

\begin{tabular}{cc}
\hline Question pair & Significance \\
\hline q1-q2 & 0,016 \\
q9-q10 & 0,034 \\
q11-q12 & 0,025 \\
q17-18 & 0,046 \\
q25-26 & 0,034 \\
q27-28 & 0,046 \\
q57-58 & 0,041 \\
\hline
\end{tabular}

Table 3 Question pair with level of significance lower than 0,05 for pairs of data related to light conditions.

\begin{tabular}{cc}
\hline Question pair & Significance \\
$\mathrm{q} 1-\mathrm{q} 33$ & 0,039 \\
\hline
\end{tabular}

Table 4 Question pairs with level of significance lower than 0,05 for pairs of data related to road type.

\begin{tabular}{cc}
\hline Question pair & Significance \\
\hline q1-q5 & 0,009 \\
q3-q7 & 0,041 \\
q52-q56 & 0,025 \\
q61-q63 & 0,046 \\
\hline
\end{tabular}

Table 5 Question pair with level of significance lower than 0,05 for pairs of data related to weather conditions.

\begin{tabular}{cc}
\hline Question pair & Significance \\
\hline q1-q17 & 0,026 \\
q3-q19 & 0,038 \\
\hline
\end{tabular}


Table 6 All question pair with level of significance lower than 0,05

\begin{tabular}{cc}
\hline Question pair & Significance \\
\hline q1-q2 & 0,016 \\
q1-q5 & 0,009 \\
q1-q17 & 0,026 \\
q1-q33 & 0,039 \\
q3-q7 & 0,041 \\
q3-q19 & 0,038 \\
q9-q10 & 0,034 \\
q11-q12 & 0,025 \\
q17-q18 & 0,046 \\
q25-q26 & 0,034 \\
q27-q28 & 0,046 \\
q52-q56 & 0,025 \\
q57-q58 & 0,041 \\
q61-q63 & 0,046 \\
\hline
\end{tabular}

The question pairs of the above table (Table 6) are these question pairs which have a level of significance lower than 0,05 according to the Wilcoxon Non-parametric Test. This significance level that is lower than 0,05 means that the variables which differ in each question of the above question pairs have a significantly different impact on the risk perception of the experts and this result will be integrated in the risk assessment algorithm.

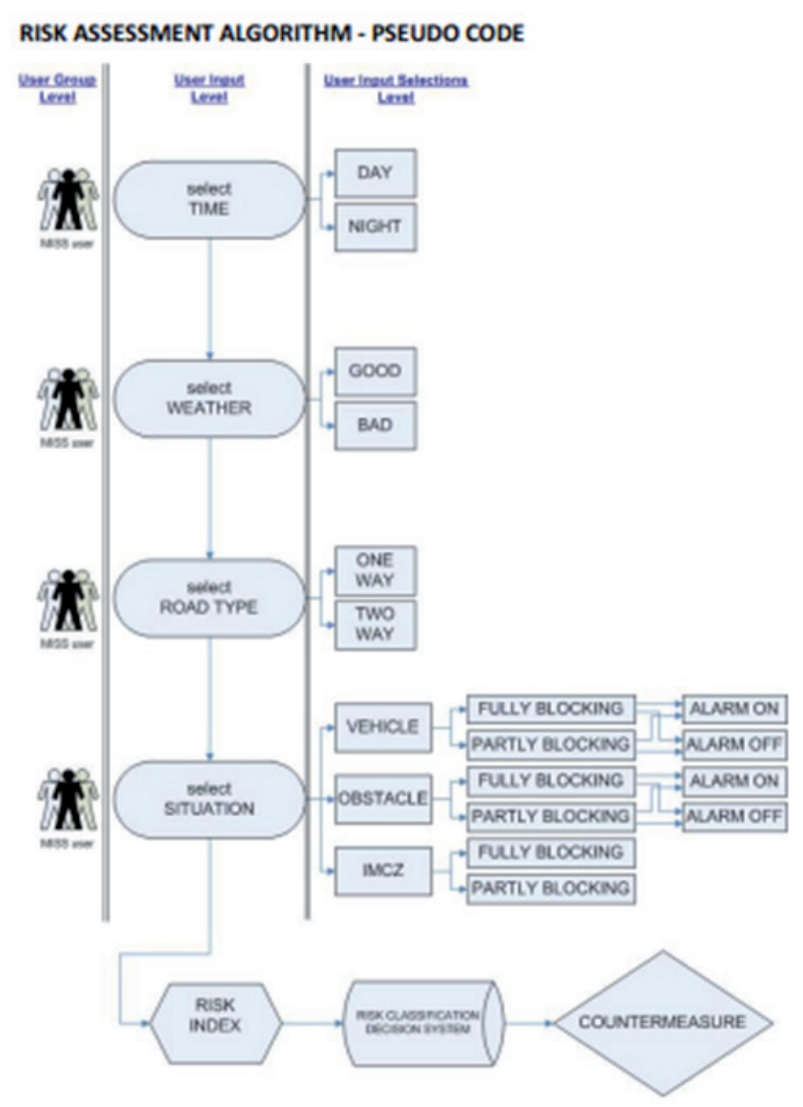

Fig. 1 Logical diagram of the MISS Risk Assessment Algorithm

\section{General description of the algorithm}

4 Categories, 14 different variables (1a-4h, as shown below) can be combined with each other, generating 64 possible combinations.

1- Time - Daylight (1a) - Night (1b)

2- Weather - Good (2a) - Bad (2b)

3- Road Type - One way (3a)- Two way (3b)

4- Situation - Vehicle - Fully blocking bus lane - Alarm lights on (4a)- Alarm lights off (4b)- Partly blocking bus lane - Alarm lights on (4c)- Alarm lights off (4d)- Obstacle - Fully blocking bus lane (4e)- Partly blocking bus lane (4f)Improperly marked construction zone - Fully blocking bus lane (4g)- Partly blocking bus lane (4h)

The user can select the corresponding variable of each category. The combination that will be formed through the selected variables will then extract the risk index which will be assigned to the selected combination. This risk index will further be interconnected with a risk classification decision system, which will easily be able to be modified by the authority or user of the risk assessment algorithm.

The results of the Wilcoxon Non-parametric test have to be integrated in the final results that the above risk assessment algorithm produces. The integration of these results is as follows: If a case (one of the questionnaire results that are indicated in Table 13, included in the risk assessment algorithm as risk index) is selected and is assigned by the final user (as described below) to a risk classification, which again is assigned to corresponding countermeasure, there has to be a distinction, which the algorithm must compute, that will separate cases according to their levels of significance. An indicative example with two cases is presented next:

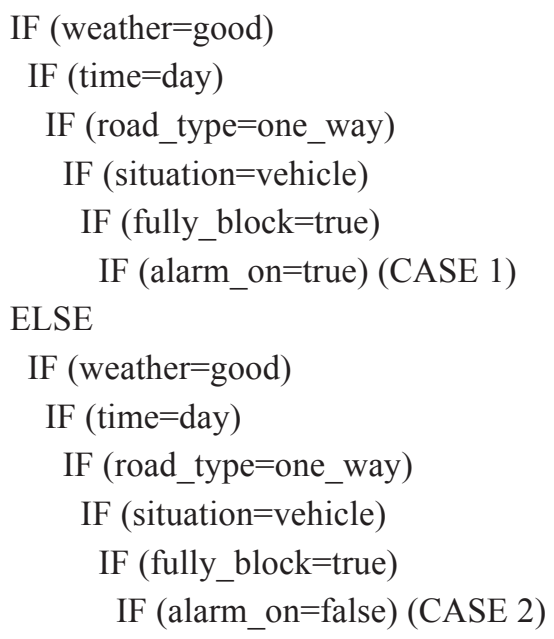

THEN ((risk classification of CASE $2=$ (risk classification of CASE 1)-1) 
Example for the extraction of the risk index from the database.

Each combination of the four variables (Time, Weather, Road type, Situation) is assigned to a numerical value which has been derived from the statistical analysis of the questionnaire (Table 4). Furthermore, the questions that have been found to differ significantly according to the Wilcoxon Non-parametric test have also been integrated in the pseudo-code of the risk assessment algorithm as cases that shall be handled separately, in case that the numerical value which has been derived from the statistical analysis of the questionnaire does not represent an initial distinction between the question pairs that are shown in Table 17. E.g. if (time=day) and (weather=good) and (road_type=One-way) and (situation=Improperly Marked Construction Zone fully blocking bus lane) then risk_index $=2,2$ E.g. if (risk_index $=2,2$ ) then (intervention=tow but not urgent) The risk indexes can be used and interpreted in two ways. First, an initial classification for the risk indexes and the corresponding countermeasure has been produced as shown below.

Table 7 Corresponding countermeasures to each risk classification and risk index domain

\begin{tabular}{ccc}
\hline Risk Index & Risk Classification & $\begin{array}{c}\text { Corresponding } \\
\text { Countermeasure }\end{array}$ \\
\hline $1,00-1,99$ & 1 & Immadiately tow \\
$2,00-2,99$ & 2 & Tow but not urgent \\
$3,00-3,99$ & 3 & Ticket \\
$4,00-5,00$ & 4 & Warning \\
\hline
\end{tabular}

Second, the classification can change and is subject of discussion as far as its final implementation is concerned. The risk classification of the risk indexes can vary according to the domain of risk indexes that will be chosen to correspond to each risk class. An example is shown in the following table.

Table 8 Alternative solution for the corresponding countermeasures to each risk classification and risk index domain

\begin{tabular}{ccc}
\hline Risk Index & $\begin{array}{c}\text { Risk } \\
\text { Classification }\end{array}$ & Corresponding Countermeasure \\
\hline $1,00-1,75$ & 1 & Immediately tow \\
$1,76-2,50$ & 2 & Tow but not urgent \\
$2,51-3,25$ & 3 & Ticket \\
$3,26-4,00$ & 4 & Warning \\
$4,01-5,00$ & 5 & Ignore (Do nothing) \\
\hline
\end{tabular}

Implementation of the Risk Assessment Algorithm.

The Risk Assessment Algorithm has been implemented in HTML code. The user has to make four selections. The first selection is concerned with the Time selection, the second is concerned with the Weather conditions selection, the third is concerned with the Road Type and the fourth is concerned with the description of the Situation. After these selections the user must press the Submit button, or alternatively the user can submit the Reset button and re-select everything. After the selection of the submit button the HTML code computes the Risk Index and provides alternatively a table with the risk classification or the corresponding countermeasures. In case that the user does not select one or more variables need for the computation of the risk index by the algorithm, then the algorithm shows an error message and directs the user to a re-selection.

\section{Acknowledgement}

Research has been supported by the MISS (Monitor Integrated Safety System) research project funded under the 6th Framework Program of the European Union.

\section{References}

Afwahlberg, A. E. (2002) Characteristics of low speed accidents with buses in public transport. Accident Analysis \& Prevention. 34 (5). pp. 637-647. DOI: $10.1016 / \mathrm{s} 0001-4575(01) 00063-\mathrm{x}$

Afwahlberg, A. E. (2004) Characteristics of low speed accidents with buses in public transport: part II. Accident Analysis \& Prevention. 36 (1) pp. 63-71.

DOI: $10.1016 / \mathrm{s} 0001-4575(02) 00128-8$

Albertsson, P., Falkmer, T. (2005) Is there a pattern in European bus and coach incidents? A literature analysis with special focus on injury causation and injury mechanisms. Accident Analysis \& Prevention. 37 (2). pp. 225-233.

DOI: 10.1016/j.aap.2004.03.006

Mirza, S. Mirza, M., Chotani, H., Luby, S. (1999) Risky behavior of bus commuters and bus drivers in Karachi, Pakistan. Accident Analysis \& Prevention. 31 (4). pp. 329-333. DOI: $10.1016 / \mathrm{s} 0001-4575(98) 00025-6$ 